\title{
BIANALYTIC FUNCTIONS WITH EXCEPTIONAL VALUES
}

\author{
P. KRAJKIEWICZ
}

\begin{abstract}
Let $f(z, \bar{z})$ be a bianalytic function which omits the value zero in some deleted neighborhood $A$ of an isolated singularity $z_{0}$. It is shown that there is a function $g(z)$ analytic on $A$ and a function $h(z, \bar{z})$ bianalytic on $A$ with a nonessential singularity at $z_{0}$ such that $f(z, \bar{z})=g(z) h(z, \bar{z})$ on $A$.
\end{abstract}

A function $f(z, \bar{z})$ is said to be polyentire or $(n+1)$ entire if and only if there exist $(n+1) \geqq 1$ entire functions $f_{0}(z), f_{1}(z), \cdots, f_{n}(z)$ such that $f(z, \bar{z})=f_{0}(z)+\bar{z} f_{1}(z)+\bar{z}^{2} f_{2}(z)+\cdots+\bar{z}^{n} f_{n}(z)$, where $\bar{z}$ denotes the complex conjugate of $z$. This function $f(z, \bar{z})$ is said to be bientire if and only if $n=1$ in the above expression for $f(z, \bar{z})$. In [1], M. B. Balk considered the problem of characterizing those polyentire functions which have a bounded set of zeros. The author, by utilizing $\mathrm{H}$. Cartan's extension of the Nevanlinna theory of meromorphic functions to linear combinations of entire functions [3], succeeded in establishing the following elegant result.

THEOREM 1. If a polyentire function $f(z, \bar{z})$ is never zero in some neighborhood of $\infty$, then there exists an entire function $g(z)$ and there exists a polynomial $P(z, \bar{z})$ in $z$ and $\bar{z}$ such that

$$
f(z, \bar{z})=P(z, \bar{z}) e^{g(z)} .
$$

The above result suggests the more general problem of obtaining an analogous characterization for polyanalytic functions which are never zero in some neighborhood of an isolated singularity, finite or infinite. In this paper we succeed in obtaining such a characterization for the class of bianalytic functions by appealing to the theory of quasi normal families of analytic functions [4]. As an immediate application we obtain a particularly simple proof of Theorem 1 in the case of bientire functions. As a further application we obtain a new demonstration of Picard's big theorem for bianalytic functions [2].

Received by the editors June 9, 1972.

AMS (MOS) subject classifications (1969). Primary 3081; Secondary 3002, 3061.

Key words and phrases. Bianalytic functions, polyanalytic functions, isolated singularity, exceptional values, Picard theorem, quasi normal families.

(C) American Mathematical Society 1973 
For purposes of completeness we give several definitions. A function $f(z, \bar{z})$ is said to be polyanalytic or $(n+1)$ analytic on some nonempty subset $A$ of the finite complex plane $\Gamma$ if and only if there exist $(n+1) \geqq 1$ functions $f_{0}(z), f_{1}(z), \cdots, f_{n}(z)$ analytic on $A$ such that

$$
f(z, \bar{z})=\sum_{k=0}^{n} \bar{z}^{k} f_{k}(z),
$$

for all $z \in A$, where $\bar{z}$ denotes the complex conjugate of $z$. This function $f(z, \bar{z})$ is said to be bianalytic on $A$ if and only if $n=1$ in the above expression for $f(z, \bar{z})$.

We now need the following uniqueness result.

Lemma 1. Let $f(z, \bar{z})$ be polyanalytic on an open subset $A$ of $\Gamma$ and suppose that $f(z, \bar{z})$ is represented on $A$ by equation (1). Then the functions $f_{0}(z), f_{1}(z), \cdots, f_{n}(z)$ are uniquely determined on $A$ by $f(z, \bar{z})$.

Proof. It suffices to show that if $f(z, \bar{z}) \equiv 0$ on $A$, then $f_{k}(z) \equiv 0$ on $A$ for $k=0,1, \cdots, n$. If we introduce the operator $\partial / \partial \bar{z}=(\partial / \partial x+i \partial / \partial y) / 2$, then it is easy to verify that $\partial / \partial \bar{z} f(z, \bar{z})=\sum k \bar{z}^{k-1} f_{k}(z)$ for $z \in A$. Thus if $f(z, \bar{z}) \equiv 0$ on $A$, then $\partial^{k} / \partial \bar{z}^{k} f(z, \bar{z}) \equiv 0$ on $A$ for $k=0,1, \cdots, n$. From the above $(n+1) \geqq 1$ equations we deduce that $f_{k} \equiv 0$ on $A$ for $k=0,1, \cdots, n$. This completes the proof of Lemma 1.

Now let $f(z, \bar{z})$ be polyanalytic on a subset $A$ of $\Gamma$ and suppose that $f(z, \bar{z})$ is represented on $A$ by equation (1). Let $z_{0}$ be an arbitrary complex number, finite or infinite. Then $z_{0}$ is termed an isolated singularity of $f(z, \bar{z})$ if and only if there is some neighborhood $N$ of $z_{0}$ so that $N-$ $\left\{z_{0}\right\} \subseteq A$. In view of Lemma 1 , the following definition is not ambiguous. A point $z_{0}$ is termed an essential isolated singularity of $f(z, \bar{z})$ if and only if $z_{0}$ is an isolated singularity of $f(z, \bar{z})$ and $z_{0}$ is an essential isolated singularity of at least one of the functions $f_{0}(z), f_{1}(z), \cdots, f_{n}(z)$.

Now let $f(z, \bar{z})$ be polyanalytic with an isolated singularity $z_{0}$. A finite complex number $\lambda$ is said to be an exceptional value for $f(z, \bar{z})$ at $z_{0}$ if and only if there is some neighborhood $N$ of $z_{0}$ such that $f(z, \bar{z})$ is defined on $N-\left\{z_{0}\right\}$ and $f(z, \bar{z})-\lambda$ never vanishes on $N-\left\{z_{0}\right\}$.

To simplify the discussion to follow we introduce some notation. If $z_{0} \in$ $\Gamma$ and $0<R \leqq+\infty$, we define $A\left(z_{0}, R\right)$ to be all $z \in \Gamma$ with $0<\left|z-z_{0}\right| \leqq R$. If $z_{0}=\infty$ and $0 \leqq R<+\infty$, we define $A\left(z_{0}, R\right)$ to be all $z \in \Gamma$ with $|z| \geqq R$. Next if $0<\rho<+\infty$ and if $f$ is a complex valued function which is continuous and never zero on $|z|=\rho$, we define $\Delta_{\rho} f$ to be $1 / 2 \pi$ times the change in the argument of $f$ on the positively oriented circumference $|z|=\rho$. Finally if $f(z, \bar{z})$ is polyanalytic on $A(\infty, R)$ and is represented on $A(\infty, R)$ by equation (1), then for $R \leqq \rho<+\infty$, we introduce the auxiliary 
function $f_{\rho}(z)$ defined by the condition that

$$
f_{\rho}(z)=\sum_{k=0}^{n} \rho^{2 k} z^{n-k} f_{k}(z),
$$

for $z \in A(\infty, R)$. Observe that $f_{\rho}(z)$ is analytic on $A(\infty, R)$ and that $f_{\rho}(z)=$ $z^{n} f(z, \bar{z})$ for $|z|=\rho \geqq R$.

We now need the following result.

LEMMA 2. Let $f(z, \bar{z})$ be polyanalytic on $A(\infty, R)$ and represented on $A(\infty, R)$ by equation (1) where $n \geqq 1$ and $f_{n}(z) \not \equiv 0$ on $A(\infty, R)$. If $f(z, \bar{z})$ has an exceptional value at $\infty$, then $f_{n}(z)$ has the exceptional value zero at $\infty$.

Proof. It suffices to consider the case when $f(z, \bar{z})$ has the exceptional value zero at $\infty$. There is some $R \leqq R_{1}$ so that $f(z, \bar{z}) \neq 0$ for $z \in A\left(\infty, R_{1}\right)$. Hence there is some integer $p$ so that $\Delta_{\rho} f(z, \bar{z})=p$ for all $\rho \geqq R_{1}$. From equation (2) we see that $\Delta_{\rho} f_{\rho}(z)=p+n$ for all $\rho \geqq R_{1}$. Let $\sigma>R_{1}$ be such that $f_{n}(z) \neq 0$ for $|z|=\sigma$. Now $f_{\rho}(z) / \rho^{2 n} \rightarrow f_{n}(z)$ as $\rho \rightarrow+\infty$ uniformly with respect to $z$ on $|z|=\sigma$. Hence there is some $\rho_{0}>\sigma$ so that $\Delta_{\sigma} f_{\rho}(z)=$ $\Delta_{\sigma} f_{\rho}(z) / \rho^{2 n}=\Delta_{\sigma} f_{n}(z)$ for all $\rho \geqq \rho_{0}$. Thus if $\rho \geqq \rho_{0}$, we see that $\Delta_{\sigma} f_{n}(z)=$ $\Delta_{\sigma} f_{\rho}(z) \leqq \Delta_{\rho} f_{\rho}(z)=p+n$. Thus if $\sigma>R_{1}$ is such that $f_{n}(z) \neq 0$ for $|z|=\sigma$, it then follows that $\Delta_{\sigma} f_{n}(z) \leqq p+n$. It therefore follows that there is some $R_{2}>R_{1}$ so that $f_{n}(z) \neq 0$ for $z \in A\left(\infty, R_{2}\right)$. Hence zero is an exceptional value for $f_{n}(z)$ at $\infty$. This completes the proof of the lemma.

We are now in a position to establish our main result.

THEOREM 2. Let $f(z, \bar{z})$ be bianalytic on $A\left(z_{0}, R\right)$ and admit the exceptional value zero at $z_{0}$. Then there exists a function $g(z)$ which is analytic and never zero on $A\left(z_{0}, R\right)$ and there exists a function $h(z, \bar{z})$ which is bianalytic on $A\left(z_{0}, R\right)$ and which does not have an essential singularity at $z_{0}$ such that

for all $z \in A\left(z_{0}, R\right)$.

$$
f(z, \bar{z})=h(z, \bar{z}) g(z),
$$

Proof. It suffices to consider the case when $z_{0}=\infty$. Let $f(z, \bar{z})$ be represented on $A(\infty, R)$ by equation (1) where $n=1$. It suffices to consider only the case when $f_{0}(z) \not \equiv 0$ and $f_{1}(z) \not \equiv 0$ on $A(\infty, R)$. By Lemma 2 , there is some $R_{1} \geqq R$ so that $f(z, \bar{z}) \neq 0$ and $f_{1}(z) \neq 0$ for $z \in A\left(\infty, R_{1}\right)$. Hence there exist integers $p$ and $q$ so that $\Delta_{\rho} f(z, \bar{z})=p$ and $\Delta_{\rho} f_{1}(z)=q$ for all $\rho \geqq R_{1}$. From equation (2) we see that $\Delta_{\rho} f_{\rho}(z)=p+1$ for all $\rho \geqq R_{1}$. Let $\lambda \geqq R_{1}$ be fixed. Now $f_{1}(z) \neq 0$ for $|z|=\lambda$ and $f_{\rho}(z) / \rho^{2} \rightarrow f_{1}(z)$ as $\rho \rightarrow+\infty$ uniformly with respect to $z$ on $|z|=\lambda$. Hence there is some $\mu>\lambda$ so that $\Delta_{\lambda} f_{\rho}(z) / \rho^{2}=\Delta_{\lambda} f_{1}(z)=q$ for all $\rho \geqq \mu$. Hence $\Delta_{\lambda} f_{\rho}(z)=q$ for all $\rho \geqq \mu$. Thus in summary we see that $\Delta_{\lambda} f_{\rho}(z)=q$ and $\Delta_{\rho} f_{\rho}(z)=p+1$ whenever $\rho \geqq \mu$. Consequently $\Delta_{\lambda} f_{\rho}(z) / f_{1}(z)=0$ and $\Delta_{\rho} f_{\rho}(z) / f_{1}(z)=p+1-q=r$ whenever $\rho \geqq \mu$. Thus for $\rho \geqq \mu$, we see that the equation $z f_{0}(z) / f_{1}(z)=-\rho^{2}$ has 
exactly $r$ solutions, counting multiplicities, in $\lambda \leqq|z| \leqq \rho$. Consequently if $\sigma \geqq \rho \geqq \mu$, we see that the equation $z f_{0}(\rho z) / \rho f_{1}(\rho z)=-(\sigma / \rho)^{2}$, has exactly $r$ solutions in $\lambda / \rho \leqq|z| \leqq \sigma / \rho$. If in the above equation we first let $\sigma=\rho$ and then let $\sigma=\sqrt{ } 2 \rho$ we deduce that if $\rho \geqq \mu$, then the function $z f_{0}(\rho z) / \rho f_{1}(\rho z)$ assumes each of the values -1 and -2 at most $r$ times in $\lambda / \mu<|z|<1$. Now let $B$ denote the annulus $\lambda / \mu<|z|<1$. Next let $\rho_{1}, \rho_{2}, \cdots$ be an increasing sequence of real numbers greater than $\mu$ and diverging to $+\infty$. Let $g_{n}(z)=$ $z f_{0}\left(\rho_{n} z\right) / \rho_{n} f_{1}\left(\rho_{n} z\right)$ for $z \in B$ and for $n=1,2, \cdots$. Let $G$ denote the collection of these functions $g_{n}(z)$ for $n=1,2, \cdots$. Since each function $g_{n}(z)$ is analytic on $B$ and assumes each of the values -1 and -2 at most $r$ times on $B$, it follows that $G$ is a quasi normal family of analytic functions on $B$ of order $s \leqq r$ [4]. We can now find some $\lambda / \mu<\delta<1$ and some subsequence $g_{n_{k}}(z)$ such that either the sequence $g_{n_{k}}(z)$ converges uniformly on $|z|=\delta$ or else the sequence diverges to $\infty$ uniformly on $|z|=\delta$. In either case we shall show that $z_{0}=\infty$ is not an essential singularity of $f_{0}(z) / f_{1}(z)$. Consider first the case when the sequence $g_{n_{k}}(z)$ converges uniformly on $|z|=\delta$. Then there is some $K>0$ so that $\left|g_{n_{k}}(z)\right|<K$ for $|z|=\delta$ and $k \geqq 1$. Hence we see that $\left|f_{0}(z) / f_{1}(z)\right|<\left(K / \delta^{2}\right)|z|$ for $|z|=\delta \rho_{n_{k}}$ and $k \geqq 1$. Hence in this case we see that $z_{0}=\infty$ is not an essential singularity of $f_{0}(z) / f_{1}(z)$. Next consider the case when the sequence $g_{n_{k}}(z)$ diverges to $\infty$ uniformly on $|z|=\delta$. Then there is some $K>1$ and some $k_{0}$ so that $\left|g_{n_{k}}(z)\right|>K$ for $|z|=\delta$ and $k \geqq k_{0}$. Consequently $\left|f_{0}(z) / f_{1}(z)\right|>\left(K / \delta^{2}\right)|z|>|z|$ for $|z|=\delta \rho_{n_{k}}$ and $k \geqq k_{0}$. Hence

$$
\Delta_{\rho} f_{0}(z) / f_{1}(z)=\Delta_{\rho}\left[f_{0}(z) / f_{1}(z)+\bar{z}\right]=\Delta_{\rho} f(z, \bar{z}) / f_{1}(z)=p-q
$$

for $\rho=\delta \rho_{n_{k}}$ and $k \geqq k_{0}$. Hence there is some $R_{2} \geqq R_{1}$ so that $f_{0}(z) / f_{1}(z) \neq 0$ for $z \in A\left(\infty, R_{2}\right)$. Hence in this case we see that $z_{0}=\infty$ is not an essential singularity of $f_{0}(z) / f_{1}(z)$. Thus in both cases we see that $z_{0}=\infty$ is not an essential singularity of $f_{0}(z) / f_{1}(z)$ as claimed. Now $f_{0}(z)$ and $f_{1}(z)$ are analytic in $|z| \geqq R$ and never zero in $|z| \geqq R_{2}$. Hence there exist polynomials $p_{0}(z)$ and $p_{1}(z)$ in $z$ and there exist functions $g_{0}(z)$ and $g_{1}(z)$ which are analytic and never zero in $|z| \geqq R$ such that $f_{0}(z)=p_{0}(z) g_{0}(z)$ and $f_{1}(z)=p_{1}(z) g_{1}(z)$ for $|z| \geqq R$. Moreover $z_{0}=\infty$ is not an essential singularity of $g_{1}(z) / g_{0}(z)$. Hence $f(z, \bar{z})=g_{0}(z)\left[p_{0}(z)+\bar{z} p_{1}(z) g_{1}(z) / g_{0}(z)\right]$ gives us the desired decomposition of $f(z, \bar{z})$. This completes the proof of Theorem 2 .

In the statement of Theorem 2, we cannot in general require that $h(z, \bar{z})$ be a polynomial in $z$ and $\bar{z}$. However if we write $h(z, \bar{z})=h_{0}(z)+\bar{z} h_{1}(z)$ we see that any one of the functions $h_{0}(z), h_{1}(z)$ may be assumed to be a polynomial in $z$.

Note that the proof of Theorem 2 gives a particularly simple demonstration of Theorem 1 in the case of bientire functions.

As a further application of Theorem 2 we have a new demonstration of Picard's big theorem for bianalytic functions [2]. 
Corollary 1. Let $f(z, \bar{z})$ be bianalytic on $A\left(z_{0}, R\right)$. If $f(z, \bar{z})$ has two distinct exceptional values at $z_{0}$, then $z_{0}$ is not an essential isolated singularity of $f(z, \bar{z})$.

Proof. We may assume that zero and one are the exceptional values for $f(z, \bar{z})$ at $z_{0}$. From Theorem 2 we see that there are functions $g_{1}(z)$ and $g_{2}(z)$ which are analytic and never zero on $A\left(z_{0}, R\right)$ and there are functions $h_{1}(z, \bar{z})$ and $h_{2}(z, \bar{z})$ which are bianalytic on $A\left(z_{0}, R\right)$ and which have a nonessential singularity at $z_{0}$ such that $f(z, \bar{z})=g_{1}(z) h_{1}(z, \bar{z})=1+g_{2}(z) h_{2}(z, \bar{z})$ for $z \in A\left(z_{0}, R\right)$. For any bianalytic function $f(z, \bar{z})=f_{0}(z)+\bar{z} f_{1}(z)$, let $f(z, w)=f_{0}(z)+w f_{1}(z)$. From Lemma 1 we see that $f(z, w)=g_{1}(z) h_{1}(z, w)=$ $1+g_{2}(z) h_{2}(z, w)$ for all $z \in A\left(z_{0}, R\right)$ and all $w \in \Gamma$. Now choose $w_{1}, w_{2} \in \Gamma$ with $w_{1} \neq w_{2}$ so that $h_{i}\left(z, w_{j}\right) \not \equiv 0$ on $A\left(z_{0}, R\right)$ for $i=1,2$ and $j=1,2$. There is some $A\left(z_{0}, R_{1}\right) \subseteq A\left(z_{0}, R\right)$ so that $h_{i}\left(z, w_{j}\right) \neq 0$ for $z \in A\left(z_{0}, R_{1}\right)$ and for $i=1,2$ and $j=1,2$. Hence $f\left(z, w_{j}\right) \neq 0,1$ for $z \in A\left(z_{0}, R_{1}\right)$ and $j=1,2$. Hence $z_{0}$ is not an essential singularity of $f\left(z, w_{j}\right)$ for $j=1,2$. Consequently $z_{0}$ is not an essential isolated singularity of $f(z, \bar{z})$. This completes the proof of the corollary.

Observe that the above result is easily seen to be valid if we adopt the following more general notion of exceptional value. Let $f(z, \bar{z})$ be bianalytic with an isolated singularity $z_{0}$. Let $\lambda(z, \bar{z})$ be bianalytic with a nonessential isolated singularity at $z_{0}$. Then $\lambda(z, \bar{z})$ is termed an exceptional value for $f(z, \bar{z})$ at $z_{0}$ if and only if zero is an exceptional value for $f(z, \bar{z})-$ $\lambda(z, \bar{z})$ at $z_{0}$. Two exceptional values $\lambda(z, \bar{z})$ and $\mu(z, \bar{z})$ for $f(z, \bar{z})$ at $z_{0}$ are said to be distinct if and only if there is some neighborhood $N$ of $z_{0}$ such that $\lambda(z, \bar{z})$ and $\mu(z, \bar{z})$ are defined on $N-\left\{z_{0}\right\}$ and $\lambda(z, \bar{z}) \not \equiv \mu(z, \bar{z})$ on $N-\left\{z_{0}\right\}$.

As a final application of Theorem 2 we mention the following result which is easily verified. Let $f(z)$ be analytic with an isolated singularity at $z_{0}$. If $\bar{z}$ is an exceptional value for $f(z)$ at $z_{0}$, then $z_{0}$ is not an essential isolated singularity of $f(z)$.

\section{REFERENCES}

1. M. B. Balk, Entire polyanalytic functions with a bounded set of zeros, Izv. Akad. Nauk Armjan. SSR Ser. Mat. 1 (1966), no. 5, 341-357. (Russian) MR 34 \#6127.

2. W. Bosch and P. Krajkiewicz, The big Picard theorem for polyanalytic functions, Proc. Amer. Math. Soc. 26 (1970). 145-150. MR 41 \#8692.

3. H. Cartan, Sur les zéroes des combinaisons linéaires de p fonctions holomorphes données, Mathematica (Cluj) 7 (1933), 5-31.

4. P. Montel, Leçons sur les familles normales de fonctions analytiques et leurs applications, Gauthier-Villars, Paris, 1927.

Department of Mathematics, University of Nebraska, Lincoln, Nebraska 68508 